\title{
Risk tables for genetic counselling in some common congenital malformations
}

\author{
CATHERINE BONAITI-PELLIE** and CHARLES SMITH \\ University Department of Human Genetics, Western General Hospital, Edinburgh EH4 $2 H U$ \\ Summary. Tables of estimated recurrence risks for some 180 specific family \\ histories are presented for three common congenital malformations. It is hoped \\ that the tables will provide standards and be useful in genetic counselling.
}

In genetic counselling, estimation of recurrence risks for the common congenital malformations depends on the empiric risks since the mode of inheritance is usually not known. However, the recurrence risk in any particular family depends on the family history, the sex of affected individuals, on the severity of the disease in the family, and on several other factors. The object of this paper is to describe the derivation of a set of tables of recurrence risks estimated from a multifactorial model of liability to the malformation (Falconer, 1965/1966). It is hoped that these tables will be useful to clinicians and provide standards for genetic counselling in these disorders.

\section{Material}

To estimate recurrence risks with the multifactorial model two statistics are required, namely the frequency of the condition in the population and the frequency in first-degree relatives of affected individuals. Data on these for three common congenital malformations in England are given in Table I. Estimates of the correlation in liability between first-degree relatives can be read from Fig. 1 in Smith (1970/1971) or derived from formula (3) in Reich, James, and Morris (1972/1973).

A method to derive the recurrence risks with the multifactorial model has been given by Smith (1971a). Information about affected first-, second-, and thirddegree relatives and about unaffected relatives can be included. The method can handle differences in frequency between sexes and take account of different severity-age classes. The computer program RISKMF (Smith, 1972) is used to derive the risk estimates. Details of family history are given, listing for each person

Received 27 April 1974.

* Stagiaire de recherches, INSERM (Unité de Recherche de Génétique Médicale, Hôpital des Enfants Malades, Paris, France). the sex, disease status, and relationship to the person at tisk.

The number of possible family histories that could be considered had to be limited. Preliminary trials showed that inclusion of unaffected second- and third-degree relatives was not important in affecting the recurrence risks. Moreover, for first-degree relatives, the change in reeurrence risk with $n$ normal relatives was approximately $n$ times the change for one normal relative. Thus a wide variety of families with $n$ normal relatives could be covered by extrapolation. Similarly, the sex of affected $\vec{\varphi}$ second and third degree relatives did not affect the risis very much, so could be ignored. By contrast, the rists were influenced to a much greater extent by whether affected individuals were on one or on both sides of the pedigree, so this was taken into account.

\section{Results}

The estimated recurrence risks for the three congenital malformations in Table I are given in Tables $\exists$ II, III, and IV; these tables are in the form of output by the computer. Each table lists the mal-? formation studied, the author, country and date of the report, the frequency for each sex and for sexese combined, and the estimated correlation in liability between first-degree relatives. There are four main columns, for the four possible parental types, and 27 rows for the various family histories within parental type. Each main column is separated into two secondary columns giving the risks with a $D$ paternal or with a maternal relative affected.

As an example of extrapolation to other families $N$ consider a family with cleft lip \pm cleft palate (Fig. 1). The nearest situation is line 11 , Table II, with a 0 risk of $21.6 \%$ (ignoring the paternal affected rela- $\omega$ tive). The existence of two unaffected sibs lowers the risk by $2 \times 2.2 \%$ (line 12-line 13), while theo existence of the affected paternal relative increases $\mathbb{\bigotimes}$ 
TABLE I

FREQUENCIES OF THREE COMMON MALFORMATIONS IN ENGLAND, AND ESTIMATES OF THE CORRELATION IN LIABILITY AMONG FIRST-DEGREE RELATIVES

\begin{tabular}{|c|c|c|c|c|c|c|}
\hline \multirow{2}{*}{ Malformation } & \multirow{2}{*}{ Author } & \multicolumn{3}{|c|}{ Population Frequency ( $\%)$} & \multirow{2}{*}{$\begin{array}{c}\text { Frequency in } \\
\text { First-degree } \\
\text { Relatives } \\
(\%)\end{array}$} & \multirow{2}{*}{$\begin{array}{l}\text { Correlation } \\
\text { in Liability }\end{array}$} \\
\hline & & All & Males & Females & & \\
\hline Pyloric stenosis & Carter and Evans (1969) & 0.30 & 0.50 & $0 \cdot 10$ & $4 \cdot 0(S, O, P)$ & $0.37 \pm 0.013$ \\
\hline Anencephaly and spina bifida & Carter and Evans (1973) & $0 \cdot 29$ & $0 \cdot 21$ & $0 \cdot 38$ & $4 \cdot 4(S)$ & $0.39 \pm 0.019$ \\
\hline
\end{tabular}

$S=$ sibs $; O=$ offspring $; P=$ parents.

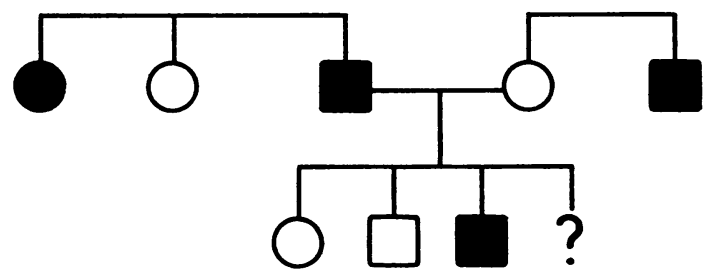

FIG. 1.

TABLE II

RECURRENCE RISKS (\%) FOR MULTIFACTORIAL INHERITANCE Cleft lip ( + or - ) cleft palate in England (Carter, 1965; 1969)

\begin{tabular}{|c|c|c|c|c|c|c|c|c|}
\hline & \multicolumn{8}{|c|}{ Parents } \\
\hline & \multicolumn{2}{|c|}{ Neither Affected } & \multicolumn{2}{|c|}{ Father Affected } & \multicolumn{2}{|c|}{ Mother Affected } & \multicolumn{2}{|c|}{ Both Affected } \\
\hline & Pat & Mat & Pat & Mat & Pat & Mat & Pat & Mat \\
\hline $\begin{array}{l}\text { No sibs } \\
1 \text { sib U }\end{array}$ & \multicolumn{2}{|c|}{$\begin{array}{l}0 \cdot 1 \\
0 \cdot 1\end{array}$} & \multicolumn{2}{|c|}{$\begin{array}{l}3 \cdot 0 \\
2 \cdot 8\end{array}$} & \multicolumn{2}{|c|}{$\begin{array}{l}3 \cdot 5 \\
3 \cdot 2 \\
\end{array}$} & \multicolumn{2}{|c|}{$\begin{array}{l}35 \cdot 4 \\
32 \cdot 3\end{array}$} \\
\hline $\begin{array}{l}1 \mathrm{M} \operatorname{sib} A \\
1 \mathrm{~F} \operatorname{sib} A \\
1 \mathrm{~F} \operatorname{sib} A+1 \operatorname{sib} U \\
\end{array}$ & \multicolumn{2}{|c|}{$\begin{array}{l}2 \cdot 6 \\
3 \cdot 1 \\
2 \cdot 9\end{array}$} & \multicolumn{2}{|c|}{$\begin{array}{r}10.0 \\
10.9 \\
9.8\end{array}$} & \multicolumn{2}{|c|}{$\begin{array}{l}10.8 \\
11.8 \\
10.6\end{array}$} & \multicolumn{2}{|c|}{$\begin{array}{l}40 \cdot 8 \\
41 \cdot 6 \\
38 \cdot 9\end{array}$} \\
\hline $\begin{array}{l}2 M \text { sibs } A \\
1 M+1 F \text { sibs } A \\
2 F \text { sibs } A \\
2 F \text { sibs } A+1 \text { sib } U\end{array}$ & \multicolumn{2}{|c|}{$\begin{array}{l}8 \cdot 1 \\
8 \cdot 8 \\
9 \cdot 5 \\
8 \cdot 8\end{array}$} & \multicolumn{2}{|c|}{$\begin{array}{l}18 \cdot 1 \\
19 \cdot 1 \\
20 \cdot 1 \\
18 \cdot 2\end{array}$} & \multicolumn{2}{|c|}{$\begin{array}{l}18 \cdot 9 \\
19 \cdot 9 \\
20 \cdot 9 \\
18 \cdot 9\end{array}$} & \multicolumn{2}{|c|}{$\begin{array}{l}44 \cdot 4 \\
45 \cdot 1 \\
45 \cdot 7 \\
43 \cdot 1\end{array}$} \\
\hline $\begin{array}{l}1 \text { 2nd-degree relative } A \\
1 M \text { sib } 1 \text { 2nd } A \\
1 \mathrm{~F} \text { sib } 1 \text { 2nd } A \\
1 \text { F sib } 1 \text { 2nd } A+1 \text { sib } U \\
2 \text { sibs }+1 \text { 2nd } A \\
2 \text { sibs }+1 \text { 2nd } A+1 \text { sib } U\end{array}$ & $\begin{array}{l}0 \cdot 6 \\
5 \cdot 2 \\
5 \cdot 9 \\
5 \cdot 4 \\
13 \cdot 1 \\
11 \cdot 9\end{array}$ & $\begin{array}{l}0 \cdot 7 \\
5 \cdot 3 \\
6 \cdot 1 \\
5 \cdot 6 \\
13 \cdot 3 \\
12 \cdot 2\end{array}$ & $\begin{array}{l}3 \cdot 8 \\
11 \cdot 4 \\
12 \cdot 4 \\
11 \cdot 1 \\
21 \cdot 0 \\
19 \cdot 1\end{array}$ & $\begin{array}{l}12 \cdot 4 \\
21 \cdot 6 \\
22 \cdot 7 \\
20 \cdot 5 \\
29 \cdot 6 \\
27 \cdot 3\end{array}$ & $\begin{array}{l}13 \cdot 6 \\
22 \cdot 8 \\
23 \cdot 9 \\
21 \cdot 6 \\
30 \cdot 3 \\
28 \cdot 1\end{array}$ & $\begin{array}{l}4 \cdot 4 \\
12 \cdot 1 \\
13 \cdot 1 \\
11 \cdot 8 \\
21 \cdot 5 \\
19 \cdot 6\end{array}$ & $\begin{array}{l}40 \cdot 0 \\
44 \cdot 7 \\
45 \cdot 4 \\
42 \cdot 6 \\
48 \cdot 6 \\
45 \cdot 6\end{array}$ & $\begin{array}{l}40 \cdot 0 \\
44 \cdot 2 \\
44 \cdot 9 \\
42 \cdot 1 \\
48 \cdot 0 \\
45 \cdot 1\end{array}$ \\
\hline \multirow[t]{2}{*}{ 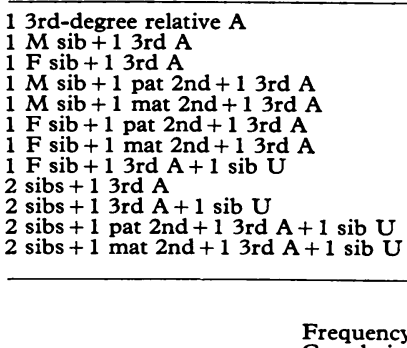 } & $\begin{array}{l}0 \cdot 3 \\
3 \cdot 8 \\
4 \cdot 4 \\
6 \cdot 3 \\
7 \cdot 9 \\
7 \cdot 1 \\
8 \cdot 7 \\
4 \cdot 1 \\
11 \cdot 2 \\
10 \cdot 3 \\
13 \cdot 4 \\
15 \cdot 2\end{array}$ & $\begin{array}{r}0 \cdot 3 \\
3 \cdot 9 \\
4 \cdot 5 \\
7 \cdot 8 \\
6 \cdot 6 \\
8 \cdot 6 \\
7 \cdot 4 \\
4 \cdot 2 \\
11 \cdot 4 \\
10 \cdot 4 \\
15 \cdot 1 \\
13 \cdot 8\end{array}$ & $\begin{array}{l}3 \cdot 6 \\
11 \cdot 0 \\
11 \cdot 9 \\
12 \cdot 3 \\
23 \cdot 4 \\
13 \cdot 3 \\
24 \cdot 6 \\
10 \cdot 7 \\
20 \cdot 5 \\
18 \cdot 6 \\
19 \cdot 9 \\
28 \cdot 9\end{array}$ & $\begin{array}{r}6 \cdot 4 \\
15 \cdot 0 \\
16 \cdot 0 \\
17 \cdot 0 \\
26 \cdot 4 \\
18 \cdot 1 \\
27 \cdot 6 \\
14 \cdot 3 \\
24 \cdot 4 \\
22 \cdot 2 \\
24 \cdot 0 \\
31 \cdot 0\end{array}$ & $\begin{array}{r}7 \cdot 3 \\
16 \cdot 0 \\
17 \cdot 1 \\
27 \cdot 5 \\
17 \cdot 8 \\
28 \cdot 6 \\
18 \cdot 9 \\
15 \cdot 3 \\
25 \cdot 0 \\
22 \cdot 9 \\
31 \cdot 8 \\
24 \cdot 5\end{array}$ & $\begin{array}{r}4 \cdot 1 \\
11.7 \\
12.7 \\
24.4 \\
12.9 \\
25.6 \\
13.9 \\
11.4 \\
21 \cdot 1 \\
19.2 \\
29.5 \\
20.3\end{array}$ & $\begin{array}{l}38 \cdot 7 \\
43 \cdot 5 \\
44 \cdot 3 \\
47 \cdot 2 \\
47 \cdot 0 \\
47 \cdot 9 \\
47 \cdot 7 \\
41 \cdot 4 \\
47 \cdot 5 \\
44 \cdot 6 \\
47 \cdot 8 \\
47 \cdot 6\end{array}$ & $\begin{array}{l}38 \cdot 8 \\
43 \cdot 2 \\
43 \cdot 9 \\
47 \cdot 2 \\
46 \cdot 3 \\
47 \cdot 9 \\
47 \cdot 0 \\
41 \cdot 2 \\
47 \cdot 1 \\
44 \cdot 3 \\
47 \cdot 8 \\
47 \cdot 0\end{array}$ \\
\hline & egree & s (\%) & $\begin{array}{c}\text { Both Sexes } \\
0 \cdot 10 \\
42 \cdot 0\end{array}$ & \multicolumn{2}{|c|}{$\begin{array}{c}\text { Males } \\
0.13 \\
42 \cdot 0\end{array}$} & $\begin{array}{c}\text { Females } \\
0 \cdot 07 \\
42 \cdot 0\end{array}$ & & \\
\hline
\end{tabular}

$\mathbf{M}=$ male $; \mathbf{F}=$ female $; A=$ affected $\mathbf{U}=$ unaffected pat = paternal ; mat = maternal 
TABLE III

RECURRENCE RISKS (\%) FOR MULTIFACTORIAL INHERITANCE

Congenital pyloric stenosis in England (Carter and Evans, 1969)

\begin{tabular}{|c|c|c|c|c|c|c|c|c|}
\hline & \multicolumn{8}{|c|}{ Parents } \\
\hline & \multicolumn{2}{|c|}{ Neither Affected } & \multicolumn{2}{|c|}{ Father Affected } & \multicolumn{2}{|c|}{ Mother Affected } & \multicolumn{2}{|c|}{ Both Affected } \\
\hline & Pat & Mat & Pat & Mat & Pat & Mat & Pat & Mat \\
\hline $\begin{array}{l}\text { No sibs } \\
1 \text { sib U }\end{array}$ & \multicolumn{2}{|c|}{$\begin{array}{l}0.3 \\
0.3\end{array}$} & \multicolumn{2}{|c|}{$\begin{array}{l}3 \cdot 7 \\
3 \cdot 4\end{array}$} & \multicolumn{2}{|c|}{$\begin{array}{l}5 \cdot 1 \\
4 \cdot 7 \\
\end{array}$} & \multicolumn{2}{|c|}{$\begin{array}{l}29 \cdot 8 \\
27 \cdot 1\end{array}$} \\
\hline $\begin{array}{l}1 \mathrm{M} \operatorname{sib} A \\
1 \mathrm{~F} \operatorname{sib} A \\
1 \mathrm{~F} \operatorname{sib} A+1 \operatorname{sib} U\end{array}$ & \multicolumn{2}{|c|}{$\begin{array}{l}3 \cdot 2 \\
4 \cdot 6 \\
4 \cdot 3\end{array}$} & \multicolumn{2}{|c|}{$\begin{array}{l}10 \cdot 2 \\
12 \cdot 8 \\
11 \cdot 6\end{array}$} & \multicolumn{2}{|c|}{$\begin{array}{l}12 \cdot 1 \\
14 \cdot 8 \\
13 \cdot 5\end{array}$} & \multicolumn{2}{|c|}{$\begin{array}{l}35 \cdot 3 \\
37 \cdot 8 \\
34 \cdot 9\end{array}$} \\
\hline $\begin{array}{l}2 \mathrm{M} \text { sibs } \mathrm{A} \\
1 \mathrm{M}+1 \mathrm{~F} \text { sibs } \mathrm{A} \\
2 \mathrm{~F} \text { sibs } \mathrm{A} \\
2 \mathrm{~F} \text { sibs } \mathrm{A}+1 \text { sib } \mathrm{U}\end{array}$ & \multicolumn{2}{|c|}{$\begin{array}{r}8 \cdot 6 \\
10 \cdot 7 \\
13 \cdot 0 \\
11 \cdot 9\end{array}$} & \multicolumn{2}{|c|}{$\begin{array}{l}17 \cdot 3 \\
20 \cdot 2 \\
23 \cdot 1 \\
21 \cdot 0\end{array}$} & \multicolumn{2}{|c|}{$\begin{array}{l}19 \cdot 0 \\
21 \cdot 7 \\
24 \cdot 5 \\
22 \cdot 5\end{array}$} & \multicolumn{2}{|c|}{$\begin{array}{l}39 \cdot 6 \\
42 \cdot 0 \\
44 \cdot 2 \\
41 \cdot 2\end{array}$} \\
\hline $\begin{array}{l}1 \text { 2nd-degree relative } A \\
1 \mathrm{M} \text { sib }+1 \text { 2nd } A \\
1 \mathrm{~F} \text { sib } 1 \text { 2nd } A \\
1 \mathrm{~F} \text { sib } 1 \text { 2nd } A+1 \text { sib } U \\
2 \text { sibs }+1 \text { 2nd } A \\
2 \text { sibs }+1 \text { 2nd } A+1 \text { sib } U \\
\end{array}$ & $\begin{array}{r}1 \cdot 1 \\
5 \cdot 7 \\
7 \cdot 6 \\
7 \cdot 0 \\
13 \cdot 5 \\
12 \cdot 4 \\
\end{array}$ & $\begin{array}{r}1 \cdot 2 \\
6 \cdot 1 \\
8 \cdot 1 \\
7 \cdot 5 \\
14 \cdot 1 \\
13 \cdot 0 \\
\end{array}$ & $\begin{array}{l}5 \cdot 1 \\
12 \cdot 4 \\
15 \cdot 3 \\
13 \cdot 7 \\
21 \cdot 7 \\
19 \cdot 7\end{array}$ & $\begin{array}{l}11 \cdot 0 \\
18 \cdot 8 \\
21 \cdot 9 \\
19 \cdot 8 \\
27 \cdot 6 \\
25 \cdot 1 \\
\end{array}$ & $\begin{array}{l}13 \cdot 6 \\
21 \cdot 2 \\
24 \cdot 1 \\
22 \cdot 0 \\
29 \cdot 1 \\
26 \cdot 8\end{array}$ & $\begin{array}{r}6 \cdot 7 \\
14 \cdot 1 \\
16 \cdot 9 \\
15 \cdot 3 \\
22 \cdot 9 \\
20 \cdot 9\end{array}$ & $\begin{array}{l}35 \cdot 4 \\
40 \cdot 5 \\
43 \cdot 0 \\
39 \cdot 8 \\
45 \cdot 9 \\
42 \cdot 6\end{array}$ & $\begin{array}{l}44 \cdot 9 \\
39 \cdot 6 \\
42 \cdot 0 \\
39 \cdot 0 \\
44 \cdot 8 \\
41 \cdot 7\end{array}$ \\
\hline 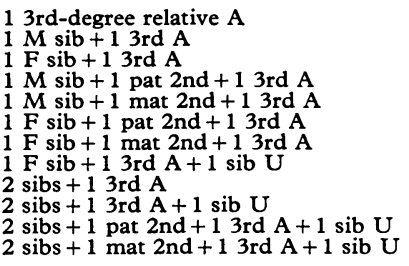 & $\begin{array}{r}0 \cdot 6 \\
4 \cdot 5 \\
6 \cdot 2 \\
7 \cdot 0 \\
8 \cdot 4 \\
9 \cdot 2 \\
10 \cdot 8 \\
5 \cdot 7 \\
11 \cdot 9 \\
11 \cdot 0 \\
14 \cdot 0 \\
15 \cdot 6\end{array}$ & $\begin{array}{r}0 \cdot 6 \\
4 \cdot 7 \\
6 \cdot 4 \\
8 \cdot 2 \\
7 \cdot 8 \\
10 \cdot 6 \\
10 \cdot 1 \\
5 \cdot 9 \\
12 \cdot 3 \\
11 \cdot 3 \\
15 \cdot 4 \\
15 \cdot 0\end{array}$ & $\begin{array}{r}4 \cdot 5 \\
11 \cdot 5 \\
14 \cdot 3 \\
13 \cdot 7 \\
20 \cdot 9 \\
16 \cdot 7 \\
24 \cdot 2 \\
12 \cdot 9 \\
20 \cdot 8 \\
18 \cdot 8 \\
21 \cdot 0 \\
27 \cdot 1\end{array}$ & $\begin{array}{r}6 \cdot 8 \\
14 \cdot 4 \\
17 \cdot 4 \\
17 \cdot 2 \\
23 \cdot 3 \\
20 \cdot 5 \\
26 \cdot 5 \\
15 \cdot 7 \\
23 \cdot 7 \\
21 \cdot 5 \\
24 \cdot 2 \\
29 \cdot 0\end{array}$ & $\begin{array}{r}8 \cdot 8 \\
16 \cdot 6 \\
19 \cdot 5 \\
25 \cdot 4 \\
19 \cdot 1 \\
28 \cdot 4 \\
22 \cdot 2 \\
17 \cdot 7 \\
25 \cdot 2 \\
23 \cdot 1 \\
30 \cdot 3 \\
25 \cdot 4\end{array}$ & $\begin{array}{c}6 \cdot 1 \\
13 \cdot 4 \\
16 \cdot 2 \\
23 \cdot 1 \\
15 \cdot 2 \\
26 \cdot 1 \\
18 \cdot 1 \\
14 \cdot 7 \\
22 \cdot 1 \\
20 \cdot 3 \\
28 \cdot 5 \\
21 \cdot 9\end{array}$ & $\begin{array}{l}33 \cdot 1 \\
38 \cdot 4 \\
41 \cdot 0 \\
43 \cdot 6 \\
42 \cdot 9 \\
46 \cdot 0 \\
45 \cdot 3 \\
37 \cdot 8 \\
44 \cdot 0 \\
40 \cdot 8 \\
45 \cdot 2 \\
44 \cdot 6\end{array}$ & $\begin{array}{l}33 \cdot 0 \\
38 \cdot 0 \\
40.5 \\
43 \cdot 4 \\
42.0 \\
45 \cdot 8 \\
44.3 \\
37 \cdot 5 \\
43.4 \\
40 \cdot 4 \\
45 \cdot 0 \\
43.7\end{array}$ \\
\hline $\begin{array}{l}\text { Frequenc } \\
\text { Correlatio }\end{array}$ & gree & $(\%)$ & $\begin{array}{c}\text { Both Sexes } \\
0.30 \\
37 \cdot 0\end{array}$ & \multicolumn{2}{|c|}{$\begin{array}{c}\text { Males } \\
0.50 \\
37 \cdot 0\end{array}$} & $\begin{array}{c}\text { Females } \\
0 \cdot 10 \\
37 \cdot 0\end{array}$ & & \\
\hline
\end{tabular}

the risk by $1.4 \%$ (line 11 -line 3 ). Putting these together gives an approximate risk of $18.6 \%$.

\section{Discussion}

The basic assumption underlying these risk estimates is that there is underlying continuous liability to the malformations. The derivation of the recurrence risks then depends on the estimated phenotypic correlation in liability among first-degree relatives. A genetic interpretation is not necessary (though the correlations among different relatives are assumed to be proportional to their degree of genetic relationship) so the methods apply to any familial disease with an underlying liability.

Other methods of estimating recurrence risks have been proposed. Morton (1969) suggested that where risks were variable between sibships, the distribution of risks may be represented by a beta distribution. Smith (1971a) compared risks estimated from the beta distribution and from the multifactorial model and found good agreement for the two models. Similarly, it is difficult in practice to discriminate between a multifactorial model and a unifactorial model with incomplete penetrance since the different modes of inheritance lead to similar frequencies in relatives (Smith, 1971b). Thus the choice of the correct model in estimating recurrence risks may not be critical, because different models lead to substantially the same risk estimates. The merits of the risk tables presented here may be that the risks are derived in a clearly defined manner and so may provide useful standards for genetic counselling. Eventually when sufficient data has accumulated on families with these congenital abnormalities, the reliability of the estimated risks can be assessed, and their accuracy further improved.

Tables have also been derived for a further eight congenital abnormalities (cleft palate, club-foot, dislocation of the hip, Hirschsprung's disease, coeliac disease, aortic stenosis, atrial septal defect, and ventricular septal defect) and are available on request. However, there is considerable variation in the frequency of all these congenital abnormalities among regions, countries, and races. Tables of risks appropriate to a particular genetic counselling 
TABLE IV

RECURRENCE RISKS (\%) FOR MULTIFACTORIAL INHERITANCE

Spina bifida + anencephaly in England (Carter and Evans, 1973)

\begin{tabular}{|c|c|c|c|c|c|c|c|c|}
\hline & \multicolumn{8}{|c|}{ Parents } \\
\hline & \multicolumn{2}{|c|}{ Neither Affected } & \multicolumn{2}{|c|}{ Father Affected } & \multicolumn{2}{|c|}{ Mother Affected } & \multicolumn{2}{|c|}{ Both Affected } \\
\hline & Pat & Mat & Pat & Mat & Pat & Mat & Pat & Mat \\
\hline $\begin{array}{l}\text { No sibs } \\
1 \text { sib U }\end{array}$ & \multicolumn{2}{|c|}{$\begin{array}{l}0.3 \\
0.3\end{array}$} & \multicolumn{2}{|c|}{$\begin{array}{r}4.9 \\
4.5 \\
\end{array}$} & \multicolumn{2}{|c|}{$\begin{array}{l}4 \cdot 3 \\
4 \cdot 0 \\
\end{array}$} & \multicolumn{2}{|c|}{$\begin{array}{l}32 \cdot 7 \\
29 \cdot 8\end{array}$} \\
\hline $\begin{array}{l}1 \mathrm{M} \operatorname{sib} \mathrm{A} \\
1 \mathrm{~F} \text { sib A } \\
1 \mathrm{~F} \text { sib } \mathrm{A}+1 \text { sib } U\end{array}$ & \multicolumn{2}{|c|}{$\begin{array}{l}4 \cdot 2 \\
3 \cdot 6 \\
3 \cdot 4 \\
\end{array}$} & \multicolumn{2}{|c|}{$\begin{array}{l}13.9 \\
12 \cdot 8 \\
11 \cdot 6\end{array}$} & \multicolumn{2}{|c|}{$\begin{array}{l}13 \cdot 0 \\
11.9 \\
10 \cdot 8\end{array}$} & \multicolumn{2}{|c|}{$\begin{array}{l}39 \cdot 3 \\
38 \cdot 4 \\
35 \cdot 4\end{array}$} \\
\hline $\begin{array}{l}2 M \text { sibs } A \\
1 M+1 F \text { sibs } A \\
2 F \text { sibs } A \\
2 F \text { sibs } A+1 \text { sib } U\end{array}$ & \multicolumn{2}{|c|}{$\begin{array}{r}11 \cdot 5 \\
10 \cdot 6 \\
9 \cdot 8 \\
9 \cdot 0\end{array}$} & \multicolumn{2}{|c|}{$\begin{array}{l}23 \cdot 0 \\
21 \cdot 8 \\
20 \cdot 6 \\
18 \cdot 8\end{array}$} & \multicolumn{2}{|c|}{$\begin{array}{l}22 \cdot 4 \\
21 \cdot 1 \\
19 \cdot 9 \\
18 \cdot 1\end{array}$} & \multicolumn{2}{|c|}{$\begin{array}{l}44 \cdot 5 \\
43 \cdot 7 \\
43 \cdot 0 \\
39 \cdot 9\end{array}$} \\
\hline $\begin{array}{l}1 \text { 2nd-degree relative } A \\
1 M \text { sib } 1 \text { 2nd } A \\
1 F \text { sib } 1 \text { 2nd } A \\
1 F \text { sib }+1 \text { 2nd } A+1 \text { sib } U \\
2 \text { sibs }+1 \text { 2nd } A \\
2 \text { sibs }+1 \text { 2nd } A+1 \text { sib } U\end{array}$ & $\begin{array}{r}1 \cdot 2 \\
7 \cdot 4 \\
6 \cdot 6 \\
6 \cdot 1 \\
14 \cdot 4 \\
13 \cdot 2 \\
\end{array}$ & $\begin{array}{r}1 \cdot 2 \\
7 \cdot 2 \\
6 \cdot 5 \\
5 \cdot 9 \\
14 \cdot 2 \\
13 \cdot 0\end{array}$ & $\begin{array}{r}6 \cdot 3 \\
15 \cdot 9 \\
14 \cdot 7 \\
13 \cdot 2 \\
23 \cdot 5 \\
21 \cdot 4 \\
\end{array}$ & $\begin{array}{l}14 \cdot 0 \\
23 \cdot 7 \\
22 \cdot 5 \\
20 \cdot 4 \\
30 \cdot 1 \\
27 \cdot 6\end{array}$ & $\begin{array}{l}12 \cdot 9 \\
22 \cdot 7 \\
21 \cdot 4 \\
19 \cdot 4 \\
29 \cdot 4 \\
26 \cdot 9\end{array}$ & $\begin{array}{r}5 \cdot 6 \\
15 \cdot 2 \\
14 \cdot 0 \\
12 \cdot 5 \\
23 \cdot 1 \\
20 \cdot 9 \\
\end{array}$ & $\begin{array}{l}37 \cdot 5 \\
43 \cdot 4 \\
42 \cdot 5 \\
39 \cdot 3 \\
47 \cdot 1 \\
43 \cdot 8\end{array}$ & $\begin{array}{l}37 \cdot 8 \\
43 \cdot 8 \\
42 \cdot 9 \\
39 \cdot 6 \\
47 \cdot 6 \\
44 \cdot 2\end{array}$ \\
\hline \multirow[t]{2}{*}{ 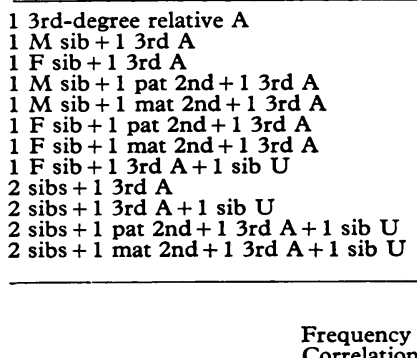 } & $\begin{array}{r}0 \cdot 6 \\
5 \cdot 9 \\
5 \cdot 2 \\
9 \cdot 1 \\
10 \cdot 1 \\
8 \cdot 2 \\
9 \cdot 1 \\
4 \cdot 8 \\
12 \cdot 8 \\
11 \cdot 7 \\
15 \cdot 0 \\
16 \cdot 0\end{array}$ & $\begin{array}{r}0 \cdot 6 \\
5 \cdot 8 \\
5 \cdot 1 \\
10 \cdot 2 \\
8 \cdot 7 \\
9 \cdot 2 \\
7 \cdot 9 \\
4 \cdot 7 \\
12 \cdot 6 \\
11 \cdot 6 \\
16 \cdot 0 \\
14 \cdot 6\end{array}$ & $\begin{array}{r}5 \cdot 8 \\
15 \cdot 2 \\
14 \cdot 1 \\
17 \cdot 2 \\
25 \cdot 8 \\
15 \cdot 9 \\
24 \cdot 5 \\
12 \cdot 7 \\
22 \cdot 9 \\
20 \cdot 8 \\
22 \cdot 5 \\
29 \cdot 4\end{array}$ & $\begin{array}{l}8 \cdot 7 \\
18 \cdot 8 \\
17 \cdot 5 \\
21 \cdot 3 \\
28 \cdot 3 \\
20 \cdot 0 \\
26 \cdot 9 \\
15 \cdot 8 \\
26 \cdot 1 \\
23 \cdot 8 \\
26 \cdot 0 \\
31 \cdot 3\end{array}$ & $\begin{array}{r}7 \cdot 8 \\
17 \cdot 9 \\
16 \cdot 6 \\
27 \cdot 2 \\
20 \cdot 6 \\
26 \cdot 0 \\
19 \cdot 2 \\
14 \cdot 9 \\
25 \cdot 5 \\
23 \cdot 1 \\
30 \cdot 6 \\
25 \cdot 6\end{array}$ & $\begin{array}{r}5 \cdot 1 \\
14 \cdot 4 \\
13.3 \\
24 \cdot 9 \\
16.5 \\
23.5 \\
15.3 \\
11.9 \\
22.3 \\
20 \cdot 2 \\
28 \cdot 8 \\
22 \cdot 1\end{array}$ & $\begin{array}{l}35 \cdot 8 \\
42.0 \\
41 \cdot 1 \\
45 \cdot 9 \\
46.6 \\
45 \cdot 1 \\
45 \cdot 8 \\
37.9 \\
45 \cdot 9 \\
42 \cdot 7 \\
46.0 \\
46.6\end{array}$ & $\begin{array}{l}35 \cdot 9 \\
42 \cdot 2 \\
41 \cdot 3 \\
46 \cdot 3 \\
46 \cdot 6 \\
45 \cdot 5 \\
45 \cdot 8 \\
38 \cdot 1 \\
46 \cdot 2 \\
42 \cdot 8 \\
46 \cdot 4 \\
46 \cdot 7\end{array}$ \\
\hline & gree & $(\%)$ & \multicolumn{2}{|c|}{$\begin{array}{c}\text { Both Sexes } \\
0 \cdot 29 \\
39 \cdot 0\end{array}$} & $\begin{array}{c}\text { Males } \\
0 \cdot 21 \\
39 \cdot 0\end{array}$ & $\begin{array}{c}\text { Females } \\
0 \cdot 38 \\
39 \cdot 0\end{array}$ & & \\
\hline
\end{tabular}

area can be produced on request (cost about $£ 10$ ) by providing us with the population frequency (two sexes) and the frequency in first degree relatives for the area concerned. For more exact risks, to include information on severity of cases or for still more complex families the program RISKMF can be used directly.

The idea of producing a set of risk tables came from Dr A. Czeizel, Hungary, who derived a set of tables of risks for sibships with congenital abnormalities from Hungarian data. We would like to thank Professor A. E. H. Emery for encouraging us to produce the Tables in the form presented here.

\section{REFERENCES}

Carter, C. O. (1965). The inheritance of common congenital malformations. Progress in Medical Genetics, 4, 59-84.
Carter, C. O. (1969). Genetics of common disorders. British Medical Bulletin, 25, 52-57.

Carter, C. O. and Evans, K. A. (1969). Inheritance of congenital pyloric stenosis. Fournal of Medical Genetics, 6, 233-254.

Carter, C. O. and Evans, K. A. (1973). Spina bifida and anencephalus in greater London. Fournal of Medical Genetics, 10, 209-234.

Falconer, D. S. (1965/1966). The inheritance of liability to certain diseases estimated from the incidence among relatives. Annals of Human Genetics, 29, 51-76.

Morton, N. E. (1969). Segregation analysis. In Computer Applications in Genetics. University of Hawaii Press, Honolulu.

Reich, T., James, J. W., and Morris, C. A. (1972/1973). The use of multiple thresholds in determining the mode of transmission of semi-continuous traits. Annals of Human Genetics, 36, 163-184.

Smith, C. (1970/1971). Heritability of liability and concordance in monozygous twins. Annals of Human Genetics, 34, 85-91.

Smith, C. (1971a). Recurrence risks for multifactorial inheritance. American fournal of Human Genetics, 23, 578-588.

Smith, C. (1971b). Discriminating between different modes of inheritance in genetic disease. Clinical Genetics, 2, 303-314.

Smith, C. (1972). Computer programme to estimate recurrence risks for multifactorial familial disease. British Medical fournal, 1, 495-497. 